\section{Ethics in Telehealth: Comparison between Guidelines and Practice-based Experience - the Case for Learning Health Systems}

\author{
Craig E. Kuziemsky ${ }^{1}$, Inga Hunter ${ }^{2}$, Shashi B. Gogia ${ }^{3}$, Sriram lyenger ${ }^{4}$, Gumindu Kulatunga ${ }^{5}$, \\ Vije Rajput ${ }^{6}$, Vignesh Subbiann ${ }^{7}, 0$ ommen John ${ }^{8}$, Araujo Kleber ${ }^{9}$, Humberto F. Mandirola ${ }^{10}$, \\ Jose Florez-Arango ${ }^{11}$, Najeeb Al-Shorbaji ${ }^{12}$, Sushil Meher ${ }^{13}$, Jai Ganesh Udayasankaran ${ }^{14}$, \\ Arindam Basu ${ }^{15}$ \\ 1 Office of Research Services and School of Business, MacEwan University, Edmonton, Alberta, \\ Canada \\ 2 School of Management, Massey University, New Zealand \\ ${ }^{3}$ Society for Administration of Telemedicine and Healthcare Informatics (SATHI), New Delhi, India \\ 4 University of Arizona College of Medicine, USA \\ 5 Postgraduate Institute of Medicine, University of Colombo, Sri Lanka \\ 6 General Practitioner, Stonydelph Health Centre, Tamworth, UK \\ 7 College of Engineering, The University of Arizona, USA \\ ${ }^{8}$ George Institute for Global Health, University of New South Wales, New Delhi, India \\ 9 NUTES Universidade Federal de Pernambuco, Brazil \\ 10 Hospital Italiano de Buenos Aires, Argentina \\ ${ }^{11}$ Texas A \& M Health Sciences Center, USA \\ ${ }^{12}$ eHealth Development Association of Jordan, Jordan \\ ${ }^{13}$ All India Institute of Medical Sciences, India \\ ${ }^{14}$ Sri Sathya Sai Central Trust, India \\ ${ }^{15}$ School of Health Sciences, University of Canterbury, New Zealand
}

issues related to technology and maintaining data security in patient-provider interactions while practitioner concern is focused on applying the guidelines to specific micro level contexts.

Conclusions: Ethics guidelines on telehealth have a macro level focus in contrast to the micro level needs of practitioners. Work is needed to close this gap. We recommend that both telehealth practitioners and ethics guideline developers better understand healthcare systems and adopt a learning health system approach that draws upon different contexts of clinical practice, innovative models of care delivery, emergent data and evidence-based outcomes. This would help develop a clearer set of priorities and guidelines for the ethical conduct of telehealth.

\section{Keywords}

Ethics, telehealth, learning health system, informatics

Yearb Med Inform 2020:44-50

http://dx.doi.org/10.1055/s-0040-1701976

\section{Introduction}

The current healthcare delivery paradigm focuses on establishing interactions across patients, providers, and settings in different contexts with ethical connotations [1]. Telemedicine is the provision of clinical care remotely, using clinical processes such as teleconsultation, telediagnosis, etc. Telehealth incorporates telemedicine but also goes beyond, with additional indirect benefits including providing preventive health support and medical education for professionals and the public [2].

Unintended consequences of telehealth usage have been reported including ethical issues [3-5]. These consequences occur due to complexities of remote care delivery by physicians, remote monitoring, and patient-provider communication through mobile health applications. Mitigating unintended consequences in telehealth practice 
should draw upon principles of medical ethics as well as best practices in informatics.

In 1803, Thomas Percival coined "medical ethics" to describe moral principles that govern the practice of medicine [6]. It was initially based on the Hippocratic Oath and included beneficence ('do good') and non-maleficence ('do no harm'). Principles of respect for autonomy (the right of a competent person to make informed decisions about her/his own medical care) and justice (especially, notions of fairness and equality) were later added to this repertory. On the other hand, codes of ethics from medical informatics address more specific guidance in informatics in addition to fundamental ethical principles. For example, both the International Medical Informatics Association (IMIA) code of ethics for health information professionals and the American Medical Informatics Association (AMIA) code of professional and ethical conduct provide ethical guidance regarding patient care, institutions and organizations, colleagues, and scientific research $[7,8]$. The principles of informatics and medical ethics, together, thus represent a core set of fundamental ideals relevant to any society, regardless of culture, and forming a system for moral reasoning and guidelines for professional ethics in medicine [9].

Other ethical frameworks, e.g., the 'CoRE-values compass and grid', incorporate important ideas and processes identified in ethical decision-making models derived from a systematic review of the literature and from published empirical evidence [10]. The National Health Services (NHS) in the United Kingdom published "A Framework for Action" on the use of data and technology within health systems. This framework includes a roadmap for moving to a whole-system, consent-based approach, which respects citizens' preferences and objections about how their personal and confidential data is used [11]. However, what needs to be examined is the inter-relationships of ethical frameworks with issues that telehealth practitioners and patients face during the day-to-day delivery of telemedicine.

The concept of learning health systems (LHSs) can potentially help with this need. The premise of a LHS is that the complex systems of people, processes, culture, policy, and technology can be continually monitored through data collection and analysis so that best practices and new knowledge can be generated and cycled back into care delivery [12]. Such an approach deviates significantly from research ethics and other frameworks that draw distinct lines between patient care and research. An LHS approach advocates for the integration of research and practice to enable high quality practice-based care. This approach can be used to understand the relationship and evolution of telehealth in different contexts of clinical practice to help us understand how to best establish a contextual fit between user needs and technology.

The goal of this paper is to provide an exploratory synthesis that compares ethical guidelines relevant to telehealth with the lived experiences of telehealth practitioners. We first identify differences between three telehealth ethical guidelines and the micro level reality of clinical practice and then discuss how these differences can be reconciled by drawing on the principles of learning health systems.

\section{Methods}

The IMIA Telehealth Working Group (WG) is made of more than 60 members from across the globe working in the telehealth arena as entrepreneurs, promoters, academics, and practitioners. During the telehealth working group meeting at Medinfo 2019 in Lyon, France, a proposal was made to develop a set of ethical guidelines on the use of telehealth for practitioners in a bid to answer some general concerns. WG members were particularly interested in comparing existing ethical guidelines with the experiences of practitioners using telehealth. After discussing different ethical issues, the WG came up with four overarching issues to use as a starting point for developing an ethical framework for telehealth.

First, telehealth-mediated interactions should facilitate patient-provider relationships. For example, digital interventions such as eCoaching can support healthy behaviours for patient groups through the monitoring and management of chronic diseases [13]. While patient-provider telehealth monitoring may achieve positive outcomes for the patient, such a process nevertheless has the potential to create undesired outcomes if patients are passively steered towards outcomes driven by an "app" rather than acting in a participatory manner [14]. Thus, there is a need to frame the boundaries between passively steering patients towards adopting practices as opposed to empowering them to become active stewards of their own care management.

Second, telehealth professionals should consider the construction, nurturing, and development of patient-provider communication via telehealth technology. For example, consider an event reported in 2019. A patient was informed of her/his imminent death by a physician using a telehealth application [15]. The patient's family and the general public responded with overwhelming outrage, particularly on social media, accusing the physician and hospital of delivering grim news with a lack of dignity. Going beyond the shortcomings by the hospital - which were duly acknowledged -, two questions arise. One, what are the ethical imperatives of such communication processes and channels? One cannot always predict the outcomes from telehealth-mediated interactions and no matter how well-intentioned, telehealth use in the context of a distance-based care cannot be value-free. Two, it begs the question of whether it is ethically appropriate for clinicians to communicate with their terminally ill patients via telemedicine. A relative of the patient who was informed over a telemedicine consultation that he was near death told a reporter that in her opinion, critically ill patients should not see a screen, rather such individuals should be seen by "a human being with compassion." [16].

Third, telehealth-mediated interactions have implications on indirect outcomes such as patient data being collected or shared inappropriately by either the patient, the provider, or other stakeholders who may be engaged within the telehealth process $[1,17]$. Issues around data integrity and security can be contentious as implications of inappropriate sharing will only be noticed downstream when significant harm may have already occurred. This calls for deliberations on the issues of data protection and privacy tailored towards telehealth applications, distinct from other data privacy and data 
sharing considerations, leading to laws such as the Health Insurance Portability and Accountability Act (HIPAA) in the United States and the Digital Information and Security in Healthcare Act (DISHA) in India $[18,19]$. Furthermore, increasing trends of incorporating artificial intelligence-based algorithms within telehealth raise new ethical challenges, including how such algorithms affect the fiduciary relationship between the patient and the provider.

Fourth, any discussion around the ethical imperative in telehealth needs to take into account that telehealth serves a range of health systems and populations with variations of age, cultures, and ethnicities within the systems. For example, privacy of patient-provider communication is especially important for telehealth clients in Asia who have medical problems associated with social stigma such as genitourinary and behavioural diseases [20]. Privacy is also a foremost concern with telehealth use by patients with mental health conditions.

We used the four issues described above to develop four open-ended questions to compare ethical guidelines for telehealth with issues from clinical practice in different care delivery contexts.

1. What cultural and regional differences impact ethical issues in telehealth?

2. What are the ethical implications of using artificial intelligence and big data generated by telemedicine services?

3. In what ways does ethics of telehealth differ from face-to-face medical practice?

4. What are the ethical issues involved in special populations? Here, we use care of elderly as an exemplar special subpopulation using telehealth services.

We used the four questions to synthesize perspectives of telehealth practitioners (practice-based evidence) and a review of telehealth ethics guidelines from three sources: (1) 2017 AMA guidelines as reported by Chaet et.al. [21], (2) the 2014 telehealth component of ethics guidelines from the Health Professions Council of South Africa [22], and (3) the 2018 World Medical Association (WMA) statement on the ethics of telemedicine [9]. We selected these three documents as exemplars of written guidelines for telehealth ethics.

\section{Results}

Our results are presented according to each of the four questions described above. The practitioner perspectives are responses from WG members and do not present a comprehensive picture of global practices nor are the findings presented in a specific order of importance.

\section{The Four Questions and Synthesis of Perspectives of Telehealth Practi- tioners and a Review of Telehealth Ethics Guidelines}

\subsection{What Cultural and Regional Differences Impact Ethical Issues in Telehealth?}

We discuss cultural and regional differences with respect to telehealth practices in four respective countries, Sri Lanka, United States, Columbia, and Argentina.

\section{A. Sri Lanka}

A medical ordinance in Sri Lanka published in 1927, Cosmetics Devices and Drugs Act no 27 of 1980, and the National Medicines Regulatory Authority Act, No. 5 of 2015 do not provide information on digital health nor do they have provisions to regulate internet-based prescriptions. The Electronic Transactions Act No. 19 of 2006, which is the common law for any electronic transaction, is currently used as the base legal act for practicing telemedicine and issuing internet-based prescriptions. Even though telemedicine continues to expand in Sri Lanka, the Sri Lankan National eHealth Guidelines and Standards document [23] published by the Sri Lanka Ministry of Health in 2016 does not have regulations on telemedicine consultations. In the absence of telehealth regulations or guidelines, the practicing physician's code of conduct is governed by routine ethical practices that ensures physicians get sufficient patient information before prescribing, obtain valid informed consent including that the patient knows the limitations of the telecare intervention, ensures privacy and confidentiality of patient information and that medical records are maintained properly.

\section{B. United States}

The Federation of United States State Medical Boards (FSMB) has prioritised telemedicine as an important medical regulatory topic that has been addressed since 2016 [24]. The Federal Council of Medicine, the regulatory and professional body, issued a new regulation for telemedicine in February 2019 but there was resistance from some physicians, and they had to revoke it, falling back to a 2001 regulation. However, in 2019, the Center for Medicare and Medicaid Services updated the Physician Fee Schedule in order to expand telehealth reimbursement services and restore the doctor-patient relationship [25]. Most recently, the Department of Veteran Affairs (DVA) in the United States implemented a federal rule that will allow providers to care for veterans across state lines, overriding any regional or state restrictions [26]. While it is not fully clear how licensure across state lines will work, and what the implications of cross state licensing may be, this new federal regulation is a step forward in increasing healthcare access to veterans and service members.

\section{Colombia}

The Colombian Ministry of Health Resolution 1448 had set an agenda for telehealth [27]. In Colombia, telehealth is used complementary to face-to-face interactions and only as an additional resource when the physical encounter is limited in some way. An informed consent is mandatory for the enrolment of patients, and telehealth delivery is mediated by a health care provider. In Colombia, the most common risks related to telehealth include loss of privacy (right of the patient), loss of confidentiality (duty of provider), and loss of data.

\section{Argentina}

In Argentina, telehealth is governed by The National Directorate of Health Information Systems under the Ministry of Health and Social Action. The overall impact on access measures, acceptability, costs, and supplier satisfaction has been positive. Argentina's eConsult services have been extended both geographically and in terms of specialized services offered. The Ministry of Health 
promotes telemedicine as a tool to expand the window of opportunity for people to access health services using eConsult [28]. However, despite the prevalence and utilisation of telehealth services, there is little uniformity around the legal frameworks, ethical guidelines or best practices needed to guide telehealth delivery in Argentina. This places the onus on clinicians to adopt ethical practices for telehealth care on a case-by-case basis.

In summary, the existing regulations on telemedicine around the world largely concentrate on limiting cross border practice. Conventionally, in all of those regulations, the physician should be registered in the country (or in some cases, the state) where the patient is situated [29]. In the United States, individual state licenses are required, requiring licenses across 50 states, the aforementioned DVA example of cross state licensing being an exception to this rule. Canada has similar problems around physician licensing across Provinces or Territories although discussions are ongoing around a national license for virtual care [30].

Such licensing practices raises the ethical question whether a professional who is approached for a remote consult can ever refuse to offer advice or services when requested. For a professional, once a financial transaction is completed, it is unethical to refuse to tender advice. On the other hand, the context of telecare makes for easy negotiation or settlement of grievance due to in-built documentation around the care process.

\subsection{What Are the Ethical Implications of Using Artificial Intelligence and Big Data Generated by Telemedicine Services?}

In face-to-face care, clinicians rely on clinical history, evaluation of signs and symptoms including special investigations (e.g. laboratory tests or diagnostic imaging) to arrive at a differential diagnosis and to plan care delivery. In telehealth, such clinical processes are constrained by time, technology, and a lack of comprehensive clinical data available for artificial intelligence-based systems. Further, there are increasing concerns about computer algorithms perpetuating existing racial and gender disparities by amplifying human biases and other risks inherent in training data [31, 32].

Synchronous telehealth consultations rely on real-time video conversations between providers and patients. Some telehealth delivery incorporates facial recognition algorithms to enable ascertaining the mood and psychological status of patients. Although facial and affective computing recognition can assist with diagnosis and treatment of mood disorders, depression, or other psychiatric illnesses, facial recognition algorithms can also result in profiling patients. Facial recognition systems can also perform sub-optimally and are at risk of reinforcing gender and racial biases, raising significant ethical issues around anonymity and confidentiality of patients who receive care through these services. Besides, as Klare argued, many telehealth systems rely on proprietary "closed source" solutions for decision or prediction algorithms, these approaches multiply risks [33]. The use of artificial intelligence (AI) in telehealth would need careful assessment of the risks from such emerging technologies. With the emerging hype around AI, this poses a risk of propagation of further inequity that already exists in some health systems.

\subsection{In What Ways Does Ethics of Tele- health Differ from Face-to-face Medical Practice?}

Telehealth lacks the in-person face-toface contact that takes place in traditional healthcare delivery. With a virtual and possibly unknown patient, physicians must try to personalize the telehealth patient as best they can. Ethical medical practice therefore remains focused on the patient as a whole person who is more than a data set or collection of digital images [34]. It is imperative that physicians ensure that they have the information they need to make well-grounded clinical decisions and diagnoses when they cannot personally conduct a physical examination, such as by having another health care professional at the patient's site when they conduct the exam, or by obtaining vital information through remote technologies.
Irrespective of the emergence of new technologies and models of care, the physicians' fundamental ethical responsibilities remain the same [35]. The responsibility rests with the healthcare providers to appreciate the difference between how fundamental responsibilities play out in the context of telehealth compared with faceto-face interactions. In as much as physicians' fundamental ethical responsibilities remain invariant, the continuum of possible patient-physician interactions in telehealth/ telemedicine gives rise to differing levels of accountability for physicians [21].

In principle, ethical issues will exist whether care delivery is via telehealth or traditional face-to-face care. These include maintaining a strong patient/client-physician/caregiver/relationship, protecting patient privacy, promoting equity in access and treatment, and seeking the best possible outcomes.

\subsection{What Are the Ethical Issues Related to Care for Elderly}

Older adults often prefer to continue living in their place of residence rather than other options such as assisted living as "aging in place" has demonstrated better health outcomes [36-39]. Monitoring an individual to detect falls or changes in health and wellbeing is an important use of telehealth that can enable older adults to live in their own home. However, ethical issues exist with telehealth support for aging in place including remote monitoring and passive data collection, empowerment of the older adult, and even limited telehealth access in some locations. Privacy and security of health information exchange between an older adult, his/her family members, and his/her healthcare providers can also be an important ethical issue as privacy restrictions may prevent a family member from accessing necessary information about his/ her family member.

While home care may enable the elderly to live longer, a key ethical question is to what extent quality of life (QoL) is improved for the patient. Mere prolonging of life without notable improvement in QoL can lead to associated suffering that affects not only patients themselves, who in some 
cases due to problems such as dementia maybe oblivious of the suffering, but also care givers and family. Further, monitoring with sensors, cameras, and similar technologies can also intrude on an individual's right to privacy, especially if imposed by well-meaning family or healthcare providers without including the older adult in the decision-making process around questions such as what to monitor, how often, and who can access the data. In 2018, a research team from Massey University sought to investigate ethical issues among 31 members of elderly population in New-Zealand using semi-structured interviews [40]. They determined 15 requirements for technology to support older adults' ageing in place. They found most of these requirements were unmet by existing commercial telehealth systems.

Access to telehealth to support care of the elderly implies either the ability to access telehealth systems via a local health care provider (e.g. getting access to specialist medical care via telehealth from a family physician), or having telehealth technology in the home. The latter can be challenging due to the digital divide and high costs of telehealth enabled care. Many telehealth systems have an initial purchase cost and then ongoing licence fees and they may also require qualified installation and ongoing technological support [37, 41, 42].

Finally, current telehealth systems tend to be 'one size fits all' and require the older adult to adapt to the technology rather than the technology fitting the context of the individual. Telehealth-enabled communication can also focus on formal health care providers while neglecting an older adults' informal support networks of family and local community [43, 44]. These informal social systems and support networks can play an important role in a person's care continuum [40].

We found three essential elements missing in the four ethical frameworks we reviewed: (1) responsible strategies that will allow for research and enable "learning" from clinical care experiences and underlying data, (2) ways to use telehealth to reduce health disparities, and (3) involving patients and care providers in the process of improving the overall telehealth system.

\section{Comparison of Ethical Guidelines and Practitioner Perspectives}

Clinicians are more concerned with direct care delivery which falls under the narrower definition of telemedicine. Ethical guidelines for telehealth have to consider the issues on a broader perspective and some are beyond the control of the clinician, which does not decrease the clinician's liability. We found differences in priorities expressed by practitioners and our analysis of ethical guidelines. While ethical guidelines and vignettes both use broad terms such as 'telehealth', 'information', and 'care', the practitioner perspective took them a step further by describing the challenges to using telehealth in a specific context, one example being how telehealth use varies across different international settings. The ethical guidelines also do not consider the fact that telehealth implementation is not a one-time event but rather takes place over a temporal dimension. To that end, the way that telehealth technologies integrate with clinical processes will develop and evolve over time and acknowledging this growth curve is an essential part of the implementation process.

\section{Discussion}

Telehealth will continue to be an essential part of healthcare delivery as patient care delivery becomes more complex and requires care coordination and information sharing over time and across providers and settings. However, telehealth introduces ethical issues due to changes in patient-provider communication patterns, access to care delivery services, and how patients interact with telehealth tools [3]. While we are often quick to criticize these new care delivery models, we must remember that healthcare is a learning health system and therefore we must learn from past experiences (both positive and negative) to improve care delivery moving forward [45]. While we always strive for positive outcomes from telehealth usage, it is not rational to assume that we can always predict, or even fully understand, how technology may change the relationship between patients, providers, and processes when implemented into complex settings [46]. Rather, we must acknowledge that unintended consequences will occur from telehealth usage and our strategy going forward must be to embrace the notion of learning health systems (LHSs) in order to better understand the new interactions and relationships that develop from telehealth usage in different contexts [1, 4, 5]. An LHS-based approach will enable us to better develop evidence-based approaches for managing unintended consequences from telehealth usage.

This paper uses a set of four discussion questions to compare telehealth guidelines from the World Medical Association, the American Medical Association, and the South African guidelines with practitioner perspectives of the IMIA Telehealth WG. We identified ethical issues that may arise from applying guidelines in the different contexts of telehealth usage and how we can use these issues to better design and manage telehealth delivery going forward.

The key finding from our work is that there is a difference in priorities expressed by practitioners and the content of ethical guidelines. While ethical guidelines and practitioner perspectives contain similar terminology such as 'telemedicine', 'information', and 'care', guidelines are more focused on the structural aspects of telehealth whereas practitioner perspective focus on behavioural challenges and implications of using telehealth in specific contexts. Practitioner perspectives provide an important source of "practice-based evidence" that helps contextualize the use of telehealth in specific settings to better understand how relationships and interactions develop between patients and providers, the ethical ramifications of using telehealth in specific contexts such as aging in place, and the challenges of integrating AI applications into actual care delivery.

Many of practitioner perspectives describe the prominent challenge to using telehealth technologies is the one size fits all nature of technology, where patients and providers are expected to adapt to the technology, rather than having a meaningful co-design between end user and technology. Research needs to continue to explore the nature of connected health delivery so we can understand the complexity of it and how to best configure the equation of 
people, processes, and technology. The gap between ethical guidelines and practitioner perspectives we identified is consistent with literature that has described the need to look at HIT implementation from micro and macro perspectives, both pre and post implementation [47]. Ethical guidelines are for the most part focused on macro dimensions of telehealth usage, which makes sense as guidelines, particularly broad guidelines such as the World Medical Association or the American Medical Association, are intended to be used across multiple settings. However, this leads to scalability issues when guidelines are implemented into micro contexts of care delivery. Guidelines also do not consider the temporal dimension of technology implementation. Implementation of HIT such as telehealth tools is a longitudinal process where we must study and learn about successes and failures to enable us to accentuate the former and address the latter. The principles of a learning health system can provide the structure to engage in the ongoing formative evaluation needed to properly develop and evaluate HIT. We also need to ask ourselves what is the value statement for patients and families from telehealth usage? While telehealth can provide patient access to services not otherwise available, it should not be used to prolong life that does not bring a notable increase in quality of life. Many of the ethical challenges from telehealth use, such as around monitoring or profiling, remain works in progress that require ongoing meaningful conversations with patients, families, and providers.

As an example, we refer back to the earlier example of the patient informed of his impending death by a physician via a telehealth application [15]. While the use of telehealth was certainly not ideal in this situation, the public reaction of excessive criticism of the physician and hospital was also inappropriate. To truly embrace the notion of a LHS we have to accept that healthcare is a complex system and HIT implementation will lead to unintended consequences. Our focus as an informatics community must then be to work together with patients, providers, policy makers, and vendors to understand and manage these consequences. We cannot just be a LHS when it suits us nor is a LHS approach intended to be a venue for unconstructive criticism of people or systems. Rather, the LHS mind set must be used to improve healthcare delivery by promoting collaboration and building meaningful relationships to enable all stakeholders to contribute to health system transformation.

The strengths of this paper are that we took a global look at ethics in telehealth and combined telemedicine guidelines with practitioner perspectives. Limitations of our approach is that we only used a selected set of guidelines and practitioners who are members of our IMIA Telehealth WG. This limits generalizability of our work. Next steps are to evaluate our findings against a larger set of guidelines and practice-based evidence.

\section{Conclusion}

The scope and importance of telehealth have increased in recent years due to the need to deliver more healthcare services across people and settings. However, a consequence of increased telehealth usage is the emergence of unintended consequences such as ethical issues. This paper compared macro level ethical guidelines for telehealth with micro level practitioner perspectives. We found that a gap exists between guidelines and practitioner perspectives, largely due to the challenges in contextualizing the use of telehealth in specific settings. We suggest a learning health system approach can help us better understand how to bridge the micro and macro dimensions of telehealth usage.

\section{Acknowledgments}

The authors would like to thank all members of the IMIA Telehealth Working Group for collectively sharing their expertise, ideas, and vision to work on this research.

\section{References}

1. Kuziemsky CE, Randell R, Borycki EM. Understanding Unintended Consequences and Health Information Technology:. Contribution from the IMIA Organizational and Social Issues Working Group. Yearb Med Inform [Internet] 2016 Nov [cited 2019 Nov 4];(1):53-60. Available from: http://dx.doi.org/10.15265/IY-2016-027

2. Gogia S. Fundamentals of Telemedicine and Telehealth. Academic Press; 2019.
3. Kuziemsky CE, Gogia SB, Househ M, Petersen C, Basu A. Balancing Health Information Exchange and Privacy Governance from a Patient-Centred Connected Health and Telehealth Perspective. Yearb Med Inform [Internet] 2018 Aug [cited 2019 Oct 31];27(1):48-54. Available from: http://dx.doi. $\mathrm{rg} / 10.1055 / \mathrm{s}-0038-1641195$

4. Coiera E, Ash J, Berg M. The unintended consequences of health information technology revisited. Yearb Med Inform [Internet] 2016 Nov [cited 2019 Nov 4];(1):163-9. Available from: http:// dx.doi.org/10.15265/IY-2016-014

5. Gogia SB, Maeder A, Mars M, Hartvigsen G, Basu A, Abbott P. Unintended Consequences of Tele Health and their Possible Solutions. Contribution of the IMIA Working Group on Telehealth. Yearb Med Inform [Internet] 2016 Nov [cited 2019 Oct 31];(1):41-6. Available from: http://dx.doi. org/10.15265/IY-2016-012

6. Pellegrino ED. Percival's Medical Ethics. Arch Intern Med [Internet] 1986 Nov [cited 2019 Oct 31];146(11):2265. Available from: http://archinte. jamanetwork.com/article.aspx?doi=10.1001/ archinte. 1986.00360230211030

7. IMIA. The IMIA code of ethics for health information professionals [Internet]. Available from: https://imia-medinfo.org/wp/wp-content/ uploads/2015/07/IMIA-Code-of-Ethics-2016.pdf

8. Petersen C, Berner ES, Embi PJ, Fultz Hollis K, Goodman KW, Koppel R, et al. AMIA's code of professional and ethical conduct 2018. J Am Med Inform Assoc [Internet] 2018 Nov 1 [cited 2020 Feb 14];25(11):1579-82. Available from: https://academic.oup.com/jamia/article $/ 25 / 11 / 1579 / 5134082$

9. World Medical Association. WMA Statement on Guiding Principles for the Use of Telehealth for the Provision of Health Care - WMA - The World Medical Association [Internet]. 2009 [cited 2019 Oct 31]. Available from: https://www.wma.net/ policies-post/wma-statement-on-guiding-principles-for-the-use-of-telehealth-for-the-provisionof-health-care/

10. Manson HM. The development of the CoRE-Values framework as an aid to ethical decision-making. Med Teach [Internet] 2012 [cited 2019 Oct 24];34(4):e258-68. Available from: http://dx.doi. org/10.3109/0142159X.2012.660217

11. Personalised health and care 2020: a framework for action [Internet]. GOV.UK. [cited 2020 Feb 14]. Available from: https://www.gov.uk/government/ publications/personalised-health-and-care-2020/ using-data-and-technology-to-transform-outcomes-for-patients-and-citizens

12. Engineering I of M and NA of. Engineering a Learning Healthcare System: A Look at the Future: Workshop Summary [Internet]. 2011 [cited 2020 Feb 14]. Available from: https://www.nap.edu/ catalog/12213/engineering-a-learning-healthcaresystem-a-look-at-the-future

13. DeJesus RS, Clark MM, Rutten LJF, Hathaway JC, Wilson PM, Link SM, et al. Wellness Coaching to Improve Lifestyle Behaviors Among Adults With Prediabetes: Patients' Experience and Perceptions to Participation. J Patient Exp 2018;5(4):314-9.

14. Adams S, Niezen M. Digital 'solutions' to un- 
healthy lifestyle 'problems': the construction of social and personal risks in the development of eCoaches. Health Risk Soc [Internet] 2016 Feb [cited 2019 Nov 3];17(7-8):530-46. Available from: https://www.tandfonline.com/doi/full/10.1 080/13698575.2015.1136409

15. Man told he's going to die by doctor on video-link robot - BBC News [Internet] [cited 2019 Nov 7]. Available from: https://www.bbc.com/news/ world-us-canada-47510038

16. Jacobs J. Doctor on Video Screen Told a Man He Was Near Death, Leaving Relatives Aghast - The New York Times [Internet] 2019 [cited 2019 Nov 3]. Available from: https://www.nytimes. com/2019/03/09/science/telemedicine-ethical-issues.html

17. Denecke K, Bamidis P, Bond C, Gabarron E, Househ M, Lau AYS, et al. Ethical issues of social media usage in healthcare. Yearb Med Inform [Internet] 2015 Aug [cited 2019 Nov 3];10(1):137-47. Available from: http://dx.doi.org/10.15265/IY2015-001

18. Ministry of Health and Family Welfare. Placing the draft of "Digital lnformation Security in Healthcare, act (DISHA)" in public domain for comments/views-reg [Internet] 2018 [cited 2020 Jan 31]. Available from: https://www.medinfo-lyon.org/en/

19. Edemekong PF, Haydel MJ. Health Insurance Portability and Accountability Act (HIPAA) [Internet] StatPearls Publishing; 2019 [cited 2020 Feb 14]. Available from: https://www.ncbi.nlm.nih.gov/ books/NBK500019/

20. Kulatunga G, Hewageegana N. Understanding the Tele-health needs of Suwásariya (Health Net) users. Indian Journal of Medical Informatics [Internet] 2014 [cited 2019 Nov 3]; Available from: https:// www.researchgate.net/profile/Dr_R_P_Pareek/publication/268213515_A_Survey_of_Image_Quality_Assessment_Techniques_for_Medical_Imaging/ links/5464b7780cf221c8f57b9b92/A-Survey-of-Image-Quality-Assessment-Techniques-for-Medical-Imaging.pdf\#page $=105$

21. Chaet D, Clearfield R, Sabin JE, Skimming K, Ethical C, on behalf of the Council on Ethical and Judicial Affairs American Medical Association. Ethical practice in Telehealth and Telemedicine. J Gen Intern Med [Internet] 2017 Oct [cited 2019 Oct 14];32(10):1136-40. Available from: http:// dx.doi.org/10.1007/s11606-017-4082-2

22. Ethics HR, Professional Practice Committee. General Ethical Guidelines for good practice in Telemedicine [Internet]. Pretoria: Health Professions Council of South Africa; 2014 [cited 2019 Oct 21]. Available from: http://www.hpcsa.co.za/

23. How Sri Lanka's Healthcare Industry Is Experimenting With Technological Solutions [Internet] [cited 2019 Nov 7]. Available from: https://roar.media/english/life/in-the-know/ how-sri-lankas-healthcare-industry-is-experimenting-with-technological-solutions/

24. United States Federation of State Medical Boards. US Medical Regulatory Trends and Actions, 2018 [Internet]. Federation of State Medical Boards; 2018 [cited 2019 Nov 6]. Available from: https:// www.fsmb.org/siteassets/advocacy/publications/ us-medical-regulatory-trends-actions.pdf

25. Physician Fee Schedule - Centers for Medicare \&
Medicaid Services [Internet] [cited 2019 Nov 6]. Available from: https://www.cms.gov/Medicare/ Medicare-Fee-for-Service-Payment/PhysicianFeeSched/

26. Office of Public and Intergovernmental Affairs. VA Expands Telehealth by Allowing Health Care Providers to Treat Patients Across State Lines [Internet] [cited $2020 \mathrm{Feb}$ 14]. Available from: https://www.va.gov/opa/pressrel/pressrelease. cfm?id=4054

27. DIJ - tienda terminos [Internet] [cited 2019 Nov 7]. Available from: https://www.minsalud.gov.co/ sites/rid/Lists/BibliotecaDigital/Forms/tienda $\% 20$ terminos.aspx?RootFolder $=\% 2$ fsites $\% 2$ frid $\%$ 2 fLists $\% 2$ fBibliotecaDigital\%2fRIDE $\% 2 \mathrm{f}$ DE\%2fDIJ\&FolderCTID=0x012000D4CDD61CAE671A41B3532ECA51ACD38E

28. Econsult [Internet] [cited 2019 Nov 6]. Available from: http://www.econsultar.com.ar/\#!/-links/.

29. Economics E. Regulatory Approaches to Telemedicine. GMC; 2018.

30. Task force launching to examine national licensure for virtual care | CMAJ News [Internet] [cited 2020 Feb 14]. Available from: https:// cmajnews.com/2019/03/26/task-force-launchingto-examine-national-licensure-for-virtual-carecmaj-109-5738/

31. Vartan S. Racial Bias Found in a Major Health Care Risk Algorithm [Internet] 2019 [cited 2019 Nov 7]. Available from: https://www.scientificamerican. com/article/racial-bias-found-in-a-major-healthcare-risk-algorithm/

32. Yu K-H, Kohane IS. Framing the challenges of artificial intelligence in medicine. BMJ Qual Saf [Internet] 2019 [cited 2019 Nov 6];28(3):238-41. Available from: http://dx.doi.org/10.1136/bmjqs-2018-008551

33. Klare BF, Burge MJ, Klontz JC, Vorder Bruegge RW, Jain AK. Face recognition performance: role of demographic information. IEEE Trans Inf Forensics Secur [Internet] 2012 Dec [cited 2019 Nov 6];7(6):1789-801. Available from: http:// ieeexplore.ieee.org/document/6327355/

34. WP Cheshire J. Telemedicine and the Ethics of Medical Care at a Distance. Ethics Med [Internet] 2017 [cited 2019 Nov 5]; Available from: http://search.proquest.com/openview/9f7705b81c9faf8d4cc0cd9e6298aac7/1?pq-origsite $=$ gscholar \& cbl $=44457 \&$ casa_token $=j R B$ V45dDgU8AAAAA:GystvwzQWYDEp-0ctT1UV1D-xoPqLmqet9iqEgrQbv7TUgcCZolG-PpMXhMUoe-cj2A2rumiZ8A

35. Mills R. AMA adopts new guidance for ethical practice in telemedicine [Internet] 2018 [cited 2019 Nov 3]. Available from: https://www.ama-assn.org/ama-adopts-new-guidance-ethical-practice-telemedicine

36. Wiles JL, Leibing A, Guberman N, Reeve J, Allen RES. The meaning of "aging in place" to older people. The Gerontologist [Internet] 2012 Jun [cited 2019 Nov 5];52(3):357-66. Available from: http://dx.doi.org/10.1093/geront/gnr098

37. Peek STM, Luijkx KG, Rijnaard MD, Nieboer ME, van der Voort CS, Aarts S, et al. Older Adults' Reasons for Using Technology while Aging in Place. Gerontology [Internet] 2016 [cited 2019 Nov 5];62(2):226-37. Available from: http:// dx.doi.org/10.1159/000430949
38. Boldy D, Grenade L, Lewin G, Karol E, Burton E. Older people's decisions regarding "ageing in place": a Western Australian case study. Australas J Ageing [Internet] 2011 Sep [cited 2019 Nov 5];30(3):136-42. Available from: http://dx.doi. org/10.1111/j.1741-6612.2010.00469.x

39. New Zealand Ministry of Health. Healthy Ageing Strategy [Internet]. New Zealand Ministry of Health; 2017 [cited 2019 Nov 5]. Available from: https://www.health.govt.nz/system/files/ documents/publications/healthy-ageing-strategy_june_2017.pdf

40. Elers P, Hunter I, Whiddett D, Lockhart C, Guesgen $\mathrm{H}$, Singh A. User requirements for technology to assist aging in place: qualitative study of older people and their informal support networks. JMIR MHealth UHealth [Internet] 2018 Jun [cited 2019 Nov 5];6(6):e10741. Available from: http://dx.doi. org/10.2196/10741

41. Kapadia V, Ariani A, Li J, Ray PK. Emerging ICT implementation issues in aged care. Int J Med Inf [Internet] 2015 Nov [cited 2019 Nov 5];84(11):892-900. Available from: http://dx.doi. org/10.1016/j.ijmedinf.2015.07.002

42. Yusif S, Soar J, Hafeez-Baig A. Older people, assistive technologies, and the barriers to adoption: A systematic review. Int J Med Inf [Internet] 2016 Jul [cited 2019 Nov 5];94:112-6. Available from: http://dx.doi.org/10.1016/j.ijmedinf.2016.07.004

43. Carretero S, Stewart J, Centeno C, Barbabella F, Schmidt A, Lamontagne-Godwin F, et al. Can technology-based services support long-term care challenges in home care? [Internet]; 2012 [cited 2019 Nov 5]; Available from: https://www. researchgate.net/profile/Francesco_Barbabella/ publication/249315045_Can_technology-based_ services_support_long-term_care_challenges_in_ home_care/links/02e7e51e460a309801000000.pdf

44. Fischer SH, David D, Crotty BH, Dierks M, Safran C. Acceptance and use of health information technology by community-dwelling elders. Int J Med Inf [Internet]. 2014 Sep [cited 2019 Nov 5];83(9):624-35. Available from: http://dx.doi. org/10.1016/j.ijmedinf.2014.06.005

45. Friedman CP, Allee NJ, Delaney BC, Flynn AJ, Silverstein JC, Sullivan K, et al. The science of Learning Health Systems: Foundations for a new journal. Learn Health Syst [Internet] 2016 [cited 2019 Nov 5]; Available from: http://doi.wiley. com/10.1002/lrh2.10020

46. Kuziemsky C, Abbas RM, Carroll N. Toward a Connected Health Delivery Framework. In: 2018 IEEE/ACM International Workshop on Software Engineering in Healthcare Systems (SEHS). IEEE; 2018. p. 46-9.

47. Kuziemsky CE. Review of social and organizational issues in health information technology. Healthc Inform Res 2015;21(3):152-60.

Correspondence to:

Arindam Basu

Room 405F

Manawa

176 St Asaph Street

Christchurch 8011, New Zealand

Tel: +64033693509

E-mail: arindam.basu@canterbury.ac.nz 